Proceedings

\title{
Forecasting Algorithms and Optimization Strategies for Building Energy Management \& Demand Response $^{\dagger}$
}

\author{
Fanlin Meng *, Kui Weng, Balsam Shallal, Xiangping Chen and Monjur Mourshed \\ School of Engineering, Cardiff University, Cardiff CF24 3AA, UK; WengK@cardiff.ac.uk (K.W.); \\ ShallalBA@cardiff.ac.uk (B.S.); Chenx82@cardiff.ac.uk (X.C.); MourshedM@cardiff.ac.uk (M.M.) \\ * Correspondence: MengF8@cardiff.ac.uk \\ † Presented at the Sustainable Places 2018 (SP 2018), Aix-les Bains 73100, France 27-29 June 2018.
}

Published: 27 August 2018

\begin{abstract}
In this paper, we look at the key forecasting algorithms and optimization strategies for the building energy management and demand response management. By conducting a combined and critical review of forecast learning algorithms and optimization models/algorithms, current research gaps and future research directions and potential technical routes are identified. To be more specific, ensemble/hybrid machine learning algorithms and deep machine learning algorithms are promising in solving challenging energy forecasting problems while large-scale and distributed optimization algorithms are the future research directions for energy optimization in the context of smart buildings and smart grids.
\end{abstract}

Keywords: building energy management; demand response; demand-side management; energy forecasting; energy optimization

\section{Introduction}

The built environment accounts for roughly $40 \%$ of all energy consumption and it is responsible for $36 \%$ of the greenhouse gas emissions in Europe. According to a public statistic dataset, the electricity consumption in the UK has increased by 17.34\% from 1990 to 2013 [1]. With the increasing demand of electricity and the penetration of electric vehicles (EVs), the traditional electricity grid experiences growing pressure in satisfying the peak demands. To alleviate such pressure, building energy management and demand response are proposed to encourage demand-side users to play a more active role to shape the energy landscape from the traditional supply-following-demand to demand-following-supply.

Demand response refers to a tariff or program designed to motivate changes in electric use by end-use customers in response to time varying prices (i.e., implicit demand response or dynamic pricing-based demand response), or to give incentive payments to customers to induce lower electricity use during the high market prices or peak demand time periods (i.e., explicit demand response or incentive-based demand response). However, to achieve efficient and reliable demand side management and demand response strategies, forecasting and optimization are critical, which gives us the motivation to research key relevant algorithms to provide important insights on the existing research gaps and the future research directions.

\section{Forecasting Algorithms}

Forecasting plays a vital role in energy supply and demand management in buildings, districts and grids. An accurate energy forecasting is essential for efficient scheduling and planning of the 
generation, distribution and use of energy [2]. The selection of forecasting methods for implementation depend on many factors such as the purpose of applications, data time resolution, data availability and update of time series [3]. In this paper, the forecasting algorithms are analyzed based on classification of building physics-based and machine learning based forecasting approaches due to their distinct model characteristics.

\subsection{Building Physics-Based Modelling}

Building physics-based approaches refer to the use of detailed physics-based equations in modelling/predicting the behaviours of the whole building and its sub-systems such as the energy consumption and indoor environmental conditions [4]. Building physics-based modelling can be further classified into the detailed comprehensive and the simplified method.

- The comprehensive method uses the principles of thermodynamics and heat-balance to calculate the disaggregated energy consumption within a building by considering weather and building fabric and system information such as building construction and their thermal properties, Heating Ventilation and Air Conditioning (HVAC) components and their operation and may also incorporate utility rate schedule as inputs [5]. The detailed comprehensive physicsbased methods of forecasting normally use building simulation engines such as EnergyPlus, ESP-r, TRANSYS, and e-QUEST.

- The simplified method is a single-measure method, such as the use of the degree-days in energy-use estimations. For instance in the ambient-temperature frequency method, it performs heating and cooling energy calculations at many different outdoor dry bulb temperature conditions, and aggregate the results by multiplying the calculation under each condition by the number of hours of occurrence of that condition [6].

\subsection{Machine Learning/Computation Intelligence Based Forecast Methods}

Although it is straightforward and easy to use, building physics-based approach always requires significant domain knowledge and is more likely to be computational intractable when the complexity of the considered problem increases (i.e., the corresponding physics-based equations become more complex). On the contrary, machine learning, which usually consists of supervised learning and unsupervised learning, does not require much domain knowledge and could learn/predict the building/sub-systems behaviours directly from history data. In this paper, machine learning based algorithms are grouped into the following categorizes.

- Supervised learning algorithms are used in the situations where the classes of data are predefined whereas the most commonly used supervised learning algorithms for energy forecasting include artificial neural networks (ANNs), random forests (RF), and support vector regressions (SVR) and deep learning algorithms [5,7].

- Unsupervised learning algorithms are often used in the situations where the classes of the data are not known in advance. In such cases, clustering can be implemented to gain insights on the data. The most commonly used clustering algorithms in energy forecasting include K-means, hierarchical clustering, and Gaussian mixture models [8].

Apart from the above analysis of different type of forecasting methods, a qualitatively comparative performances of some of building energy forecasting algorithms on computational time and modelling complexity are also shown in Figure 1. 


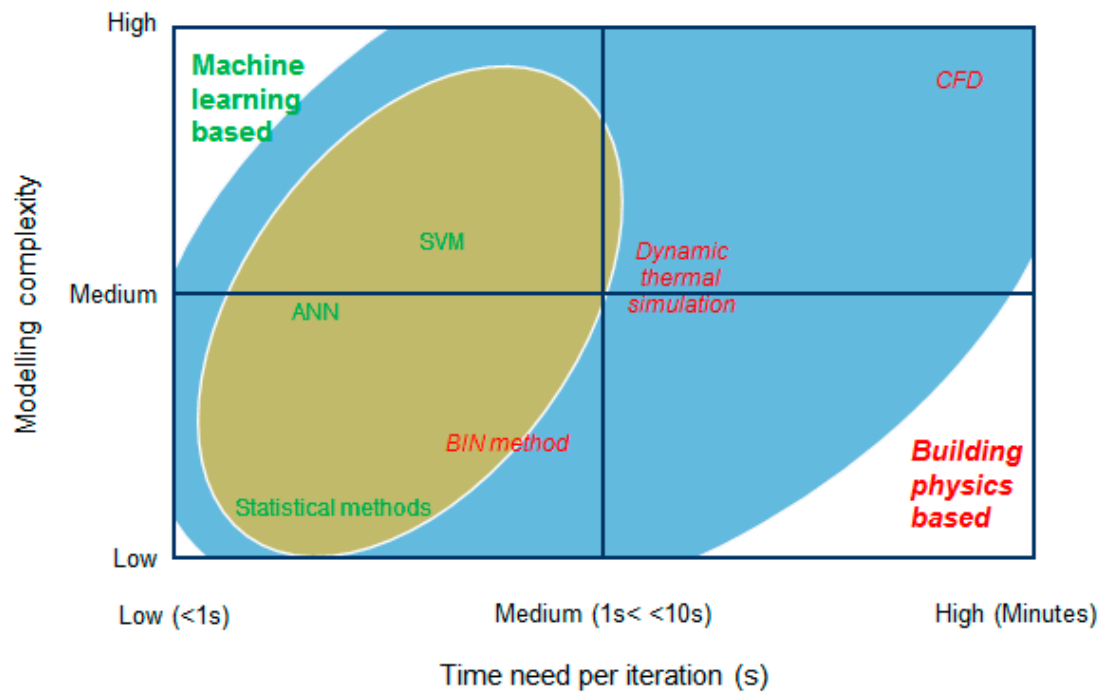

Figure 1. Comparative performances of some of building energy forecasting algorithms.

\section{Optimization Strategies}

\subsection{Optimization Models}

Based on the forecast results, energy optimization is usually needed to meet the objectives set out by the decision makers such as the business goals or the energy reduction targets. The optimization models differ according to the control objectives, decision variables, and operational constraints and can be generally categorized into the following categories:

- Multi-objective optimization [9] vs. single objective optimization where for the former there are multiple objective functions in the optimization model to be considered simultaneously whereas for the latter only one objective function is given.

- Hierarchical (multi-level optimization) [10] vs. single level optimization where for the former it considers the hierarchical interactions/game behaviors between different decision makers and usually consists of sequential decision makings whereas for single level optimization, the optimization is solved simultaneously either for a single decision maker or for multiple decision makers.

- Stochastic optimization [11] vs. deterministic optimization where for the stochastic optimization either the decision variables or the model parameters are stochastic whereas for the deterministic optimization all of them are deterministic.

\subsection{Solution Algorithms}

To solve the above optimization problems efficiently, appropriate optimization algorithms need to be developed. Depending the characteristics of the optimization problems, the solution algorithms are generally categorized into the following two groups:

- Conventional mathematical programming methods: this type of methods are usually used for well-defined optimization models with desirable mathematical properties (e.g., convex, differentiable). In such cases, exact mathematical optimization algorithms such as linear programming, convex programming, and more general nonlinear programming methods are often used to obtain optimal solutions [12].

- Metaheuristic algorithms: this type of solution algorithms are used for optimization models with ill-defined objective function/constraints or with non-convex and non-differentiable mathematical properties. These optimization problems are usually NP-hard with the computational complexity increasing exponentially with the size of the problems. In such cases, computational intelligence-based optimization algorithms (e.g., genetic algorithms, particle 
swarm optimization, simulated annealing, etc.) are usually adopted to achieve optimal/nearoptimal solutions.

\section{Discussions \& Conclusions}

Forecasting and optimization play an important part in the building energy management and demand response and also in a wider context of energy system operation and planning. In this paper we conduct a systematic and critical investigation of the current state-of-the arts with the aim to identify the current research gaps and to point out the future research directions. As such the following research gaps are identified during the above analysis in energy forecasting and optimization and potential technique routes are also suggested.

- Energy forecasting: In addition to the commonly used supervised learning and unsupervised learning methods, there are increasing research interests and trends in looking at the ensemble and hybrid machine learning algorithms [13] where the strength of each machine learning algorithm (or each type of algorithms) are combined or deep machine learning algorithms [7] to tackle challenging research problems such as the wind energy forecasting.

- Energy optimization: with the integration of demand response technologies, more and more end-users and devices will become proactive in the modern energy systems. How to manage such a tremendously large set of devices or buildings in real-time is a challenging problem for energy optimization. As such, large-scale optimization and distributed optimization techniques [14] are required in such applications. However, how to develop an efficient and scalable optimization algorithm is still an open research question.

Funding: This work has received funding from the European Union's H2020 project “Demand Response Integration Technologies: unlocking the demand response potential in the distribution grid" (DRIvE) under Grant Agreement No. 774431.

Conflicts of Interest: The authors declare no conflicts of interest.

\section{References}

1. UK Department of Energy \& Climate Change. Historical Electricity Data: 1920 to 2013; Technical Report; UK Department of Energy \& Climate Change: London, UK, 2013.

2. Iman, G.; Ardjmand, E.; Weckman, G.R.; Young, W.A. An overview of energy demand forecasting methods published in 2005-2015. Energy Syst. 2017, 8, 411-447.

3. Chirag, D.; Zhang, F.; Yang, J.; Lee, S.E.; Shah, K.W. A review on time series forecasting techniques for building energy consumption. Renew. Sustain. Energy Rev. 2017, 74, 902-924.

4. Li, X.; Jin, W. Review of building energy modeling for control and operation. Renew. Sustain. Energy Rev. 2014, 37, 517-537.

5. Zhao, H.-X.; Magoulès, F. A review on the prediction of building energy consumption. Renew. Sustain. Energy Rev. 2012, 16, 3586-3592.

6. Al-Homoud, M.S. Computer-aided building energy analysis techniques. Build. Environ. 2001, 36, 421-433.

7. Heng, S.; Xu, M.; Li, R. Deep learning for household load forecasting-A novel pooling deep RNN. IEEE Trans. Smart Grid 2017, doi:10.1109/TSG.2017.2686012.

8. Stephen, H.; Singleton, C.; Grindrod, P. Analysis and clustering of residential customers energy behavioral demand using smart meter data. IEEE Trans. Smart Grid 2016, 7, 136-144.

9. Li, D.; Chiu, W.-Y.; Sun, H.; Poor, H.V. Multiobjective optimization for demand side management program in smart grid. IEEE Trans. Ind. Inf. 2018, 14, 1482-1490.

10. Meng, F.; Zeng, X.-J.; Zhang, Y.; Dent, C.J.; Gong, D. An integrated optimization+ learning approach to optimal dynamic pricing for the retailer with multi-type customers in smart grids. Inf. Sci. 2018, 448, 215-232.

11. Zhang, Y.; Meng, F.; Wang, R.; Zhu, W.; Zeng, X.-J. A stochastic MPC based approach to integrated energy management in microgrids. Sustain. Cities Soc. 2018, 41, 349-362.

12. Deng, R.; Yang, Z.; Chow, M.-Y.; Chen, J. A survey on demand response in smart grids: Mathematical models and approaches. IEEE Trans. Ind. Inf. 2015, 11, 570-582. 
13. Ren, Y.; Suganthan, P.N.; Srikanth, N. Ensemble methods for wind and solar power forecasting-A stateof-the-art review. Renew. Sustain. Energy Rev. 2015, 50, 82-91.

14. Guo, J.; Hug, G.; Tonguz, O.K. A case for non-convex distributed optimization in large-scale power systems. IEEE Trans. Power Syst. 2017, 32, 3842-3851.

CC) (C) 2018 by the authors. Licensee MDPI, Basel, Switzerland. This article is an open access
article distributed under the terms and conditions of the Creative Commons Attribution
(CC BY) license (http://creativecommons.org/licenses/by/4.0/). 